\title{
Electron-impact recombination and excitation rates for charge-state-selected highly charged $\mathrm{Si}$ ions
}

\author{
E. Lindroth, ${ }^{1}$ I. Orban, ${ }^{1}$ S. Trotsenko, ${ }^{2}$ and R. Schuch $\oplus^{1,2, *}$ \\ ${ }^{1}$ Department of Physics, Stockholm University, AlbaNova, 10691 Stockholm, Sweden \\ ${ }^{2}$ Helmholtz Institute Jena, Fröbelstieg 3, 07743 Jena, Germany
}

(Received 6 February 2020; accepted 5 May 2020; published 11 June 2020)

\begin{abstract}
Charge-state selective recombination rate coefficients were measured by time of flight (TOF) analyzed highly charged $\mathrm{Si}$ ions extracted from an electron-beam ion trap. Additionally, the combination of simultaneous TOF and $\mathrm{x}$-ray measurements and a separation of the dielectronic recombination contribution in the $\mathrm{x}$-ray spectra is used for extracting electron-impact excitation rate coefficients for several overlaying charge states. Experimentally derived dielectronic recombination spectra for XIII and XIV Si are compared and found in excellent agreement with the results of relativistic many-body perturbation theory calculations.
\end{abstract}

DOI: 10.1103/PhysRevA.101.062706

\section{INTRODUCTION}

Highly charged ions (HCIs) and, in particular, Si ions are abundant in the universe. Recently, $40 \%$ of the baryons "missing" from the nearby universe were found as ionized intergalactic gas, absorbing in the UV spectral range [1,2]. The remaining part of the missing baryonic matter is believed to be at even higher ionization stages, absorbing in the $\mathrm{x}$ ray regime. $\mathrm{HCI}$-electron interactions are of fundamental interest and are among the most important processes in plasma [3]. Photons originating from electron-impact excitation (EIE) and electron-ion recombination have important effects on the energy balance of the plasma and provide information about distant astrophysical as well as laboratory plasmas [4]. Such data have a significant impact on guiding the modelers of the $\mathrm{x}$-ray absorption features recently observed in the intergalactic and interstellar media and offer an insight into a detailed isonuclear abundance analysis and spectroscopy of highly ionized laboratory plasmas relevant for fusion research. Electron energy-dependent EIE data of highly charged ions are particularly scarce in the literature (see, e.g., Refs. [5-7]), and accurate atomic data for this process are needed to understand and model plasmas and to extract reliable information from observed emission or absorption lines.

Important progress in obtaining accurate electron-impact data for recombination was reached in the past two decades for atomic and molecular ions of a range of charges at cooler storage rings (CSRs) [8] and for HCIs at electron-beam ion traps (EBITs) [9]. These two methods are complementary in the collision energy and use complementary detection of the electron-ion reaction products. At CSRs, predominantly the

\footnotetext{
*schuch@fysik.su.se
}

Published by the American Physical Society under the terms of the Creative Commons Attribution 4.0 International license. Further distribution of this work must maintain attribution to the author(s) and the published article's title, journal citation, and DOI. Funded by Bibsam. charge-changed ions are detected which yield predominantly total recombination data, and some cases for Si were studied this way [10-12]. Whereas, at EBITs, mostly photons are detected [13]. Important information is lost in both cases, that prohibits the disentanglement of the different processes and reaction channels. For example, if only photons are detected, the dielectronic recombination (DR) spectra of various charge states overlap and DR into high Rydberg states overlaps with the continuous as well as the resonant EIE spectra. The experiment presented here combines the detection of chargechanging processes and photon detection from an EBIT. This offers reliable and simultaneous observation of photorecombination and excitation in electron impact on several isonuclear highly charged ions.

Earlier, there were approaches to measure the chargechanging collisions in EBIT by a charge-state analysis of extracted ions [14-16] with a dipole magnet and by varying the magnetic field. It was possible to determine electron-ion cross sections or rate coefficients in these measurements [14-16]. We present an alternative approach which, through detecting the population of all charges accumulated in an EBIT and separating the extracted charges by time-of-flight (TOF) [17] together with their associated photons. The measurements give electron-ion collision rate coefficients, namely, those for DR of charge-state separated ions, and from the photons, we get also the EIE rate coefficients for a mixture of ion charge states. In fact, this method has been used by our group in measurements of DR and EIE for highly charged sulfur ions [18]. A recent publication of a theoretical approach to DR and calculations of EIE for S showed very good agreement [19] with these $S$ data [18].

In the presented paper, we report on studies of chargechanging reactions and photon emission for astrophysically relevant highly charged Si ions. From the TOF spectra, the DR rate coefficients for selected charge states are obtained, and, with their use, the separation of photon spectra due to excitation, i.e., the identification of photons without change in the charge state was performed. From there, we obtain electronion-impact excitation data that cover the energy region from the direct excitation threshold and show prominent peaks due 
to resonant excitation, i.e., resonant electron attachment and autoionization to an excited state followed by photon emission. The charge-state selected DR resonances are compared to relativistic many-body perturbation theory (RMBPT) calculations [20,21]. The up to three open-shell atomic systems in the state-of-the-art complex-scaled relativistic many-body perturbation theory in its all-order formulation that within its single- and double-excitation coupled-cluster scheme even includes some of the QED effects (radiative corrections). As a result, a comparison of DR resonance parameters (resonant energies and strengths) between theoretical predictions and experimental observations show an excellent agreement.

\section{EXPERIMENT}

In the Stockholm EBIT [17,22], an electron beam compressed by a 3-T magnetic field to a diameter of $\sim 70 \mu \mathrm{m}$ passes through a set of three drift tubes. An electrostatic trap is created by biasing the middle drift tube to a lower potential as compared to the outer electrodes. The drift tube assembly has a high positive potential with the total drift tube potential defining the energy of the electrons passing through the trap region.

Following the injection of an $\sim 10 \mathrm{~ms} \mathrm{SiH}_{4}$ gas pulse into the trap, a suitable charge-state distribution of Si ions was produced by ionizing during $900 \mathrm{~ms}$ at $8 \mathrm{kV}$. Then, a "probing" time of $310 \mathrm{~ms}$ at a given electron energy was followed by extraction of the ions at a fixed energy. The above sequence was repeated over and over again with the probing energy incremented by $3 \mathrm{eV}$ each cycle in order to cover the energy range of 1.2-2.4 keV associated with resonant excitation of a $K$-shell electron to higher shells. A schematic of these cycles for the TOF measurements is shown in Fig. 1 of Ref. [18]. $\mathrm{X}$ rays emitted during the probing time were observed with a $\mathrm{Si}(\mathrm{Li})$ detector through an aperture located at $90^{\circ}$ with respect to the electron-beam direction. Throughout the measurement, the electron current was $10 \mathrm{~mA}$, and the trap had a fixed depth of $10 \mathrm{~V}$. Following a flight path of $3 \mathrm{~m}$, the extracted ions were detected by an open electron multiplier. For each extraction, the probing energy and the digitized TOF spectrum containing the six highest charge states of Si were saved. The pressure in the EBIT tank, the extraction passage, and the drift tube, was monitored to be below $10^{-8} \mathrm{mb}$. The pressure was also varied over one order of magnitude, and an influence of the pressure variation on the ion abundances was not observed.

The earlier experiments in which extracted ions were used to derive atomic data at EBITs and an electron-beam ion source were based on slow ion extraction and magnetic separation of charge states [14-16,23]. We employ a very fast ion extraction scheme to open the electrostatic trap so fast that all ions are released within $\leqslant 100 \mathrm{~ns}$. With ejection, the emission rate of $x$ rays from the EBIT drops down to 0 . This fast extraction allows separation of charge states by TOF and yields the simultaneous detection of all charge states of the ions that leave the trap after each probing.

TOF and $\mathrm{x}$-ray spectra connected through the probing electron energies are shown in Figs. 1(a) and 1(b), respectively. Figure 1(a) shows $\mathrm{Si}^{Q+}$ ion counts in a $2 \mathrm{D}$ plot of TOF vs electron energy (charge state $Q$ is shown on the right ordinate). The width of the TOF peaks is similar to

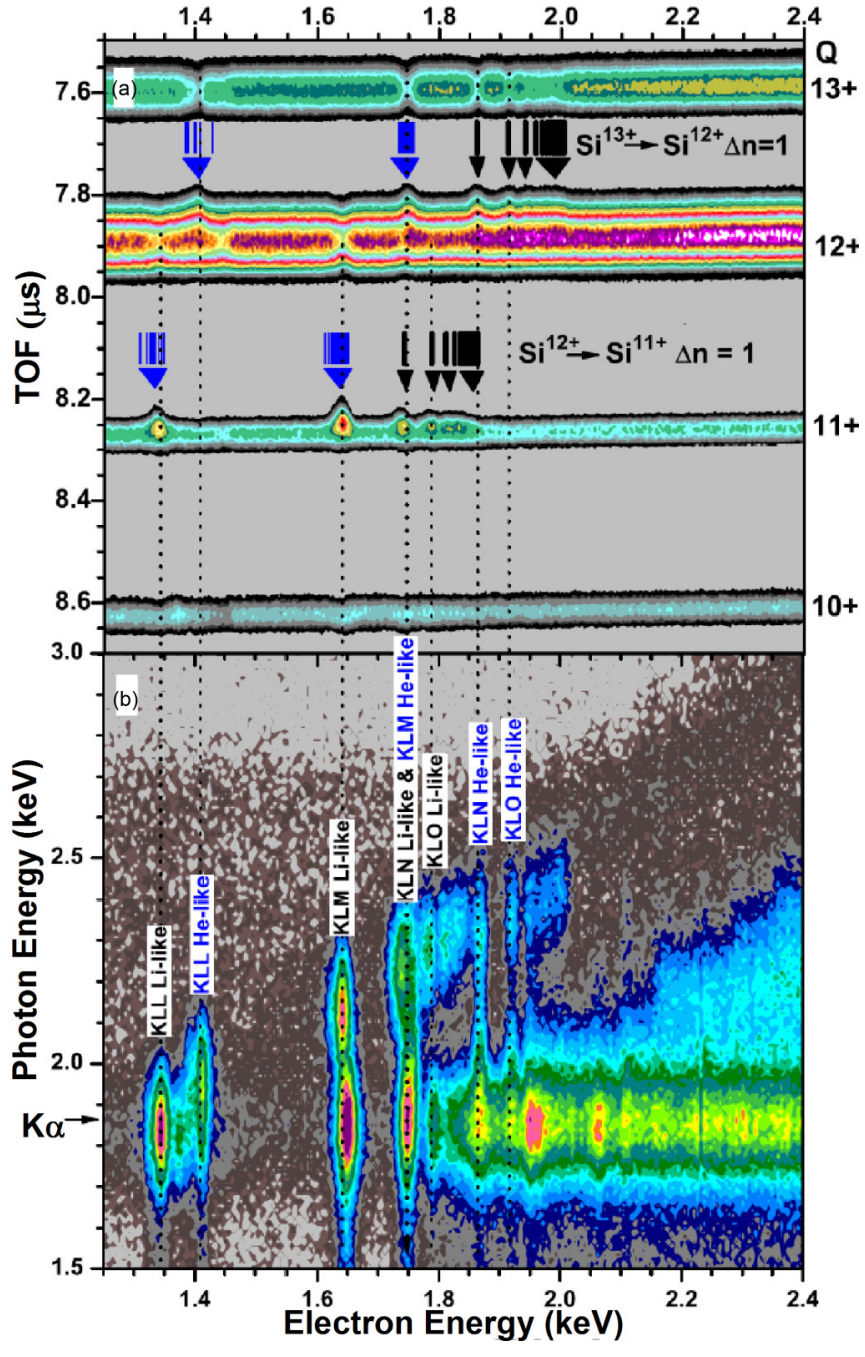

FIG. 1. (a) Ion $\mathrm{Si}^{Q+}$ counts in a two-dimensional (2D) plot of time-of-flight vs electron energy (charge state $Q$ is shown on the right ordinate). Vertical dashed lines are shown to guide the eye. The vertical bars show DR resonance positions (blue bars, RMBPT results). Arrows show the direction of DR, i.e., from a $Q+1$ charge state to a $Q$ charge state. (b) Two-dimensional contour plot of x-ray counts in photon energy vs electron energy.

the time over which the trap is opened. The vertical dashed lines are shown to guide the eye, and the vertical bars show DR resonance positions (blue bars, RMBPT results). Arrows show the direction of $\mathrm{DR}$, i.e., from a $Q+1$ charge state to a $Q$ charge state. Figure 1(b) depicts the 2D contour plot of $\mathrm{X}$-ray counts in photon energy vs electron energy. The DR resonance groups are indicated by Auger notation, according to their intermediate doubly excited states in the recombined ion. In the case of the DR resonances, the x-ray spectrum shows separately the radiative decay of the excited core and the decay of the outer electron. As an interesting example, in the lowest-energy $K L M$-type DR resonances of Li-like $\mathrm{Si}$, the excited electronic core has a triplet spin configuration, i.e., the $K$-shell (spectator) and $L$-shell (excited) electrons have parallel spins. Therefore, stabilization of the corresponding doubly excited states takes place predominantly through the decay of the outer $M$-shell electron to the single $K$-shell 
vacancy. Consequently, at these electron energies, mostly $K \beta$ photons are emitted. Toward higher electron energies in the $K L M$ resonance group, DR takes place through doubly excited states with a singlet core for which the radiative decay of the $L$-shell electron is allowed, and the associated $K \alpha$ peak becomes dominant. As a result, the maximum of the $K \beta$ peak in the $K L M$ resonance group, corresponding to the decay of the outer $M$-shell electron is located at lower electron energies as compared to the $K \alpha$ peak.

The correlated spectra offer detailed information about the processes taking place inside the EBIT. In the photon spectrum, the DR series belonging to different charge states are overlapping, and they overlap with the EIE series, hindering the extraction of recombination spectra. The TOF spectrum, on the other hand, gives selective and clean access to changes in the population of each individual charge state without the overlap occurring in the x-ray spectra. Events where the charge of the ion is not changed in the reaction are not distinguishable in the TOF counts from those where no reaction occurred. But these can be registered in the $\mathrm{x}$-ray vs electron matrix [see, e.g., Fig. 1(b)]. As Fig. 1(a) shows, at energies where DR resonances occur, the TOF spectrum contains sharp changes in the intensity of both the original and the recombined charge states. This facilitates the unambiguous identification of reaction paths, e.g., separation of EIE from DR and to make DR of $\Delta n>1$ visible. It is interesting to note that recombined ions reach the detector faster than other ions released from the trap [see, e.g., the Li-like charge state $(11+)$ where the DR peaks from recombined He-like ions are shifted to shorter TOF values]. This is probably due to space-charge effects and repelling of lower charge-state ions by the higher charge states that leave the trap earlier and these get less abundant in a DR resonance.

Comparing the charge-state distributions at the beginning and at the end of the probing time (obtained by probing for short intervals at different times) offers an overview of the charge-changing electron-ion processes taking place in the EBIT. Figure 2 shows the TOF detector counts of selected charge states as a function of electron energy. As can be seen in Fig. 2, the detected ion number shows peaks (gain of ions) and dips (loss of ions) due to charge changes by recombination. Every peak in the daughter ion corresponds to a dip in the mother ion which can be used to check the consistency in the ion transport and detection. It is known that different charge states can have somewhat different ion temperatures, hence, there could be different extraction and transport efficiencies when they travel down the drift tube. Such differences could not be observed here (e.g., by summing up the dips and peaks). We checked also the ion charge-state distribution with our $90^{\circ}$ analyzing magnet and found the same charge-state distribution as with TOF.

The nonresonant processes affecting the ion populations [radiative recombination (RR), charge exchange, evaporation of ions from the trap, etc.] produce a smooth baseline over which the effects of the resonant DR are imprinted. At energies where DR takes place, a decrease in the abundance of the recombining charge state with the simultaneous increase in the abundance of the next lower charge state is observed. As a consequence of the large differences in the abundance of neighboring charge states, even a small fraction of the ions in

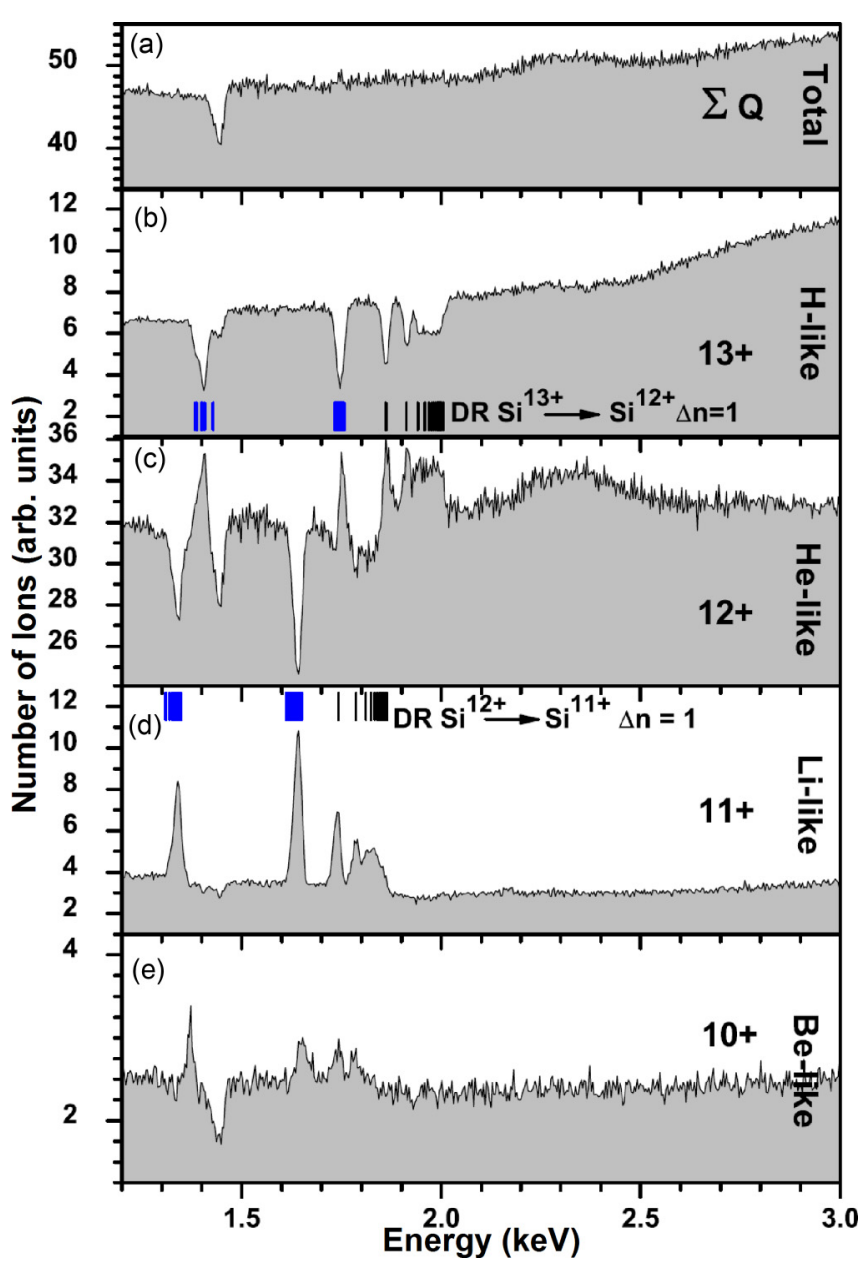

FIG. 2. TOF ion counts separated for selected $\mathrm{Si}^{Q+}$ as a function of electron energy. The vertical bars show DR resonance positions for He-like Si and Li-like Si [in boxes (b) and (d), respectively] as given in Fig. 1.

the abundant charge state undergoing DR will cause a large relative increase in the population of the less abundant ions. This is well illustrated at the Li-like DR resonance at $1.65 \mathrm{keV}$ where a change of $22 \%$ in the population of He-like ions is matched by a threefold increase in the amount of $\mathrm{Li}$-like $\mathrm{Si}$. As a proof of the linear response of the detector with the number of ions and the charge-state independent extraction efficiency, the sum of all TOF peak areas is a smooth curve in which the decrease associated with DR in the parent charge states is compensated by the increase in the resulting charge state [see Fig. 2(a)]. At $\sim 1.45 \mathrm{keV}$, the population of all charge states decreases. The dip is proportional to the amount of the ions in the respective charge state, and there is no increase in any of the neighboring charge states. We conclude that this dip is from reduced trapping of the ions in the EBIT. The dip in the trapped ion numbers disappears in the rate equation analysis as described in the next section.

\section{RESULTS AND DISCUSSIONS}

The time evolution of the charge states is described by a set of rate equations. There is an extensive literature on these 
rate equations, their solutions published [14,23-25], and we followed the general ideas of those analysis schemes. The rate equations link the population of different charge states through associated rate coefficients $\alpha$ with various indices for corresponding charge-changing processes and have as an initial condition the charge-state populations at the beginning of the probing time. The subscripts at $\alpha$ denote the charge state of the ion, whereas the superscripts, i.e., EII, DR, RR, and $C X$ represent the electron-impact ionization, dielectronic recombination, radiative recombination, and charge exchange, respectively,

$$
\begin{aligned}
\frac{d n_{Q}}{d t}= & n_{e}\left[n_{Q-1}(t) \alpha_{Q-1}^{\mathrm{EII}}-n_{Q}(t)\left(\alpha_{Q}^{\mathrm{EII}}+\alpha_{Q}^{\mathrm{DR}}+\alpha_{Q}^{\mathrm{RR}}\right)\right. \\
& \left.+n_{Q+1}(t)\left(\alpha_{Q+1}^{\mathrm{DR}}+\alpha_{Q+1}^{\mathrm{RR}}\right)\right]-n_{0}\left[n_{Q}(t) \alpha_{Q}^{C X}\right. \\
& \left.-n_{Q+1}(t) \alpha_{Q+1}^{C X}\right]-\frac{n_{Q}(t)}{\tau_{\mathrm{esc}}}
\end{aligned}
$$

In this equation $n_{Q}(t), n_{0}$, and $n_{e}$ stand for number of ions, residual gas density, and electron density, respectively. The first term in square brackets describes the breeding and depletion of ions due to electron impact ionization and recombination processes. The electron-ion interactions are described by the first term only. The second term represents the loss and gain due to charge exchange with the residual gas atoms or molecules. The last term stands for an ion escape rate from the trap. To account for overlap between electron beam and ion cloud, the first term in Eq. (1), describing electron-ion interactions is multiplied by an overlap factor $f$. Equation (1) can be written as

$$
\begin{aligned}
\frac{d n_{Q}}{d t}= & n_{Q-1}(t) f n_{e} \alpha_{Q-1}^{\mathrm{EII}} \\
& +n_{Q+1}(t) f\left[n_{e}\left(\alpha_{Q+1}^{\mathrm{DR}}+\alpha_{Q+1}^{\mathrm{RR}}\right)+\frac{n_{0} \alpha_{Q+1}^{C X}}{f}\right] \\
& -n_{Q}(t) f\left[n_{e}\left(\alpha_{Q}^{\mathrm{EII}}+\alpha_{Q}^{\mathrm{DR}}+\alpha_{Q}^{\mathrm{RR}}\right)+\frac{n_{0} \alpha_{Q}^{C X}}{f}+\frac{1}{\tau_{\mathrm{esc}} f}\right] .
\end{aligned}
$$

Ion ejection, transportation, and detection efficiencies are considered equal for neighboring charge states. A linear dependence of the detector signal on the number of ions can be checked by the peak and dip areas in adjacent charge states, and the area of the TOF peaks can be related to the number of ions by a calibration constant. However, the ion number to the TOF peak-area calibration constant appears on both sides of the rate equations (see above) and cancels out. Therefore, the peak areas can be used directly in the rate equations, and calibration of the TOF signal to the number of ions is not required. The ion intensities before and after the probing time were used as boundary conditions for solving the rate equations numerically. For the bare $\mathrm{Si}$ ions, e.g., some processes do not occur, Only RR and $C X$ feed the H-like charge state from $\mathrm{Si}^{14+}$, where $\mathrm{RR}$ is the electron energy $\left(E_{e}\right)$ dependent and $C X$ not. RR can be very well calculated for all charge states using Refs. [26,27]. RR rates for Si ions are also reported by Ref. [28] and compare well with those calculated here. The values of $n_{0} \alpha^{C X}$ are determined from an $E_{e}$-independent loss of ions that appear in the next lower charge state. $C X$ is scaled proportional to $q$ (appropriate in this narrow $q$ range) and included for all charge states. Also, a small fraction of ion loss was taken into account by $\tau_{\text {esc }}$ with those ions that do not appear in the next lower charge state.

There is a feeding term from $\mathrm{Si}^{13+}$ to $\mathrm{Si}^{14+}$ and all the following charge states as well by EII, and this rate can be taken from literature using the electron density and energy that is derived from the parameters of this experiment. The decrease in He-like $\mathrm{Si}$, e.g., is balanced by the electron-impact ionization, mostly of the $2 s$ electron in the Li-like ions. Thus, the inclusion of the EII process is necessary in all the rate equations. For $\mathrm{Si}^{11+}$, the ionization data from Ref. [29] is in the energy range of $1-3 \mathrm{keV} 4-12 \%$ higher than from Ref. [30]. The ionization data from Ref. [29] are based on the most recent measurements and calculations using the FAC code. Calculated ionization balances for Si ions are also reported by Refs. [28,31] and compare well with those of Ref. [29]. The ionization data from Ref. [30] is based on the Coulomb-Born approximation. They reported an estimated error of $40-60 \%$ in cross sections for Si ions. Therefore, the required EII cross sections are taken from Ref. [29]. The estimated error in the data analysis from this contribution is $10-15 \%$. The comparison of the EII with the feeding of $\mathrm{Si}^{14+}$ from $\mathrm{Si}^{13+}$ gives an estimate for the overlap factor $f$ in Eq. (2). And, this estimate compares well with that obtained as a function of probing energy by scaling from previous measurements [32]. We concluded from this agreement that the ion cloud diameter varied from 150 to $180 \mu \mathrm{m}$ within the given scan range, the electron-beam diameter was $70 \mu \mathrm{m}$, so the $f$ factor varied from to 0.22 to 0.15 , respectively.

With all these feeding and loss channels, a consistent description of the charge balances of the ions after probing could be obtained. So, we are quite sure that all channels influencing the charge balance were taken into account. By solving the rate equations for the different electron energies, the sum of $E_{e}$-dependent electron-ion recombination rate coefficients $\alpha_{\mathrm{RR}}+\alpha_{\mathrm{DR}}$ is obtained for the individual charge states. A continuous background due to RR is readily identified and subtracted. In fact, a comparison of the RR background rate with a calculated RR rate(see above) could be used to verify the above rate coefficient evaluation and estimated systematic error. The DR rate coefficients of Li-like, He-like, and H-like $\mathrm{Si}$ are plotted in Figs. 3(a)-3(c). We see the strong resonances of DR associated with the excitation of a $K$-shell electron to the $L$ shell during the attachment of a free electron [( $\Delta n=1)$ type DR], additionally, at higher energies $(\Delta n=2)$-type DR resonances are also observed, associated with the excitation of a $K$-shell electron to the $M$ shell.

The uncertainties in the EII (from cross sections and $f$ factor) are directly affecting the accuracy of the derived DR rate coefficients and contribute most to the total error in the absolute scale of the DR spectra for the different ions, in particular, He-like and Li-like Si. The uncertainties in the overlap between the electron beam and the ion cloud is estimated to $\sim 15 \%$. And with an error in the EII rate coefficients, we estimate the total error in the DR rate coefficients to be $22 \%$. The total error in the EIE rate coefficients is estimated to $25 \%$. The statistical errors vary from $5 \%$ for the $\mathrm{H}$ - and He-like DR to $10 \%$ for the Li-like DR. 


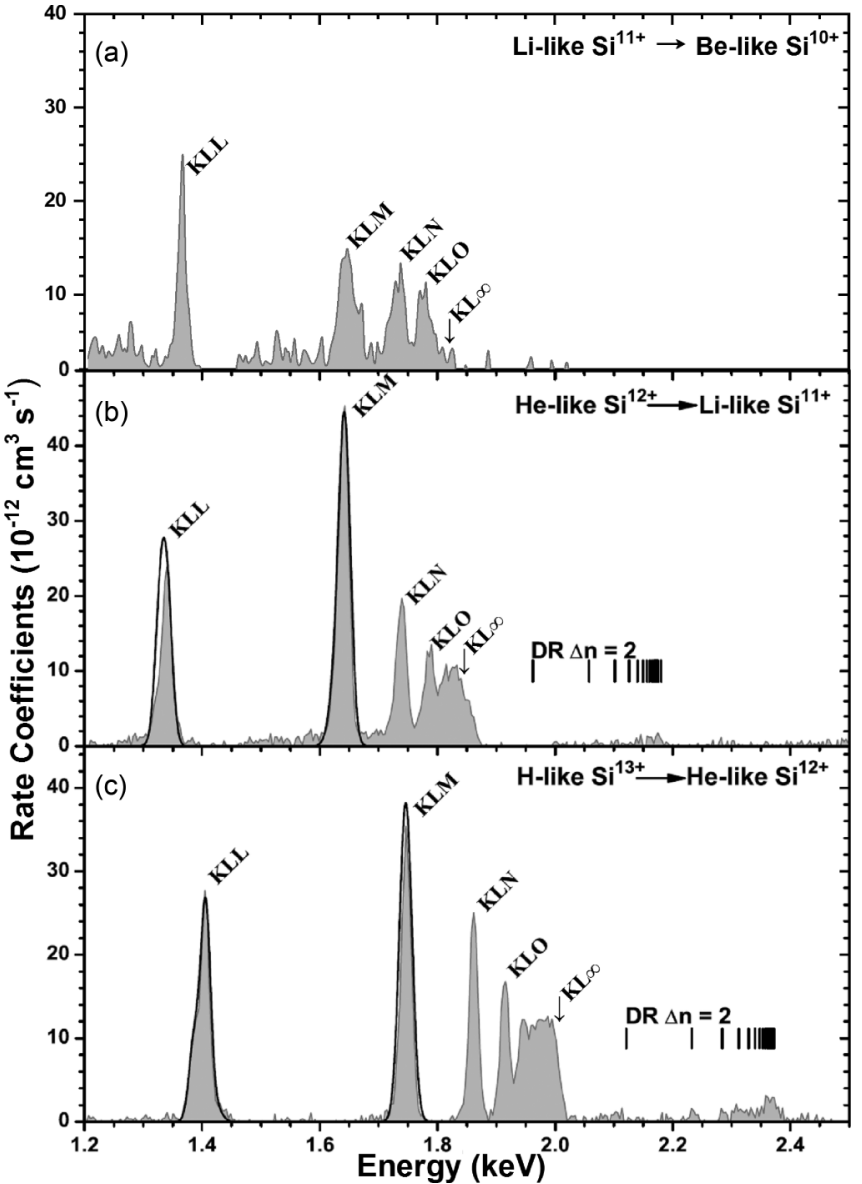

FIG. 3. Experimentally derived DR rate coefficients of (a) Lilike, (b) He-like $\mathrm{Si}$, (c) $\mathrm{H}$-like $\mathrm{Si}$, and calculated rate coefficients [solid lines in (b) and (c)]. Auger notation is used for the resolved $\Delta n=1$ resonance groups, and the vertical bars show expected positions of the $\Delta n=2 \mathrm{DR}$ resonances.

The doubly excited states of $K L L$ and $K L M$ types in $\mathrm{Si}^{12+}$ (He-like) and $\mathrm{Si}^{11+}(\mathrm{Li}$-like) have been calculated with RMBPT. These resonant states are embedded in the continuum, and we combine RMBPT with complex rotation to describe the continuum coupling and extract the Auger halfwidth as an imaginary part of a complex energy eigenvalue. For two open shells (as, e.g., resonances in He-like $\mathrm{Si}$ ), the method has been described elsewhere [21]; through an iterative procedure, the perturbation expansion of the electronelectron interaction is treated to all orders, allowing for a detailed comparison with experiment. We present the results from an extension of this method to systems with three open shells, here applied to the resonances in Li-like $\mathrm{Si}$ within second-order perturbation theory. The combination of RMPBT and complex rotation has hitherto made possible precise calculations of recombination spectra over a wide range of nuclear charges, and with this development, the method is applicable to more complex electronic structures. From the resonance positions, Auger rates and the radiative stabilization rates of the doubly excited states (obtained within the dipole approximation), we obtain the DR cross section, see, e.g., Ref. [21]. Folding it with a Gaussian resolution function with half-width $18 \mathrm{eV}$ (mostly the electron-beam

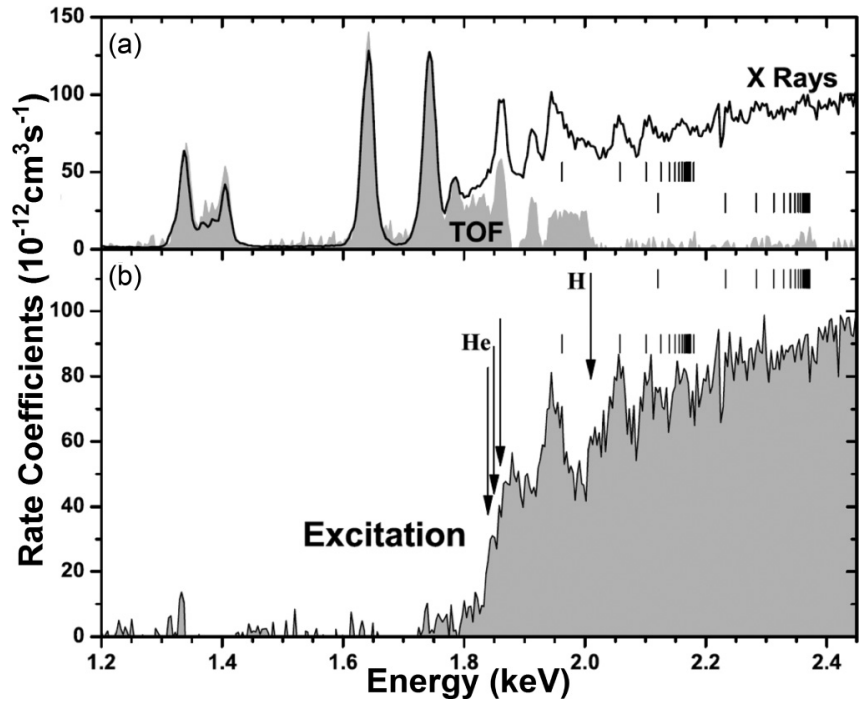

FIG. 4. (a) X rays (solid line) and mixed TOF spectra (gray area) vs electron energy. (b) $\mathrm{X}$ rays due to EIE of H-like and He-like Si (see the text) as a function of electron energy. The vertical arrows show $K \rightarrow L$ excitation thresholds in H-like (marked $\mathrm{H}$ ) and He-like $\mathrm{Si}$ (marked He). The vertical bars show the $\Delta n=2 \mathrm{DR}$ resonance positions in $\mathrm{H}$-like and He-like Si.

velocity distribution), we get the rate coefficients. These are plotted in Figs. 3(b) and 3(c) as full lines and are in excellent agreement with the experimental results in both the position of the resonances and their absolute strength.

In order to obtain EIE rate coefficients, the X-ray counts from Fig. 1(b) were projected on the electron energy axis, and the radiative recombination background was subtracted. Then, the $\mathrm{x}$-ray intensities were corrected for geometrical detection solid angle, photon absorption, and were normalized in the same way as the TOF spectra to the electron density. The systematic error from these corrections is estimated to $20 \%$. In separate $\mathrm{x}$-ray measurements, a short acquisition time (20 ms) ensured that the charge-state population during acquisition was not altered. Comparing these $\mathrm{x}$-ray spectra at different probing times, we could conclude that the amount of ions along the probing time of $310 \mathrm{~ms}$ did not change significantly. As noted earlier, the x-ray spectrum contains overlapping contributions from several charge states. This prevents the extraction of charge-state-specific photon spectra. Therefore, the X-ray curve was normalized to the number of H-like Si ions (being inside the electron beam), obtained from the $K$-RR signal of H-like to He-like Si.

To estimate and extract the fraction of photons originating from DR only, the He-like DR rate coefficient curve obtained from the TOF measurement was multiplied with 2 to account for both $\mathrm{x}$ rays emitted during the recombination of the $\mathrm{H}$-like ions. The Li-like $\mathrm{Si} \mathrm{DR}$ rate coefficient curve was multiplied with 2.7, approximately the ratio of the He-like to H-like population. The contribution from the Li-like ions was then estimated by scaling the DR part of the Be-like TOF curve shown in Fig. 2(e). Lower charge states were less abundant, and their contribution was considered insignificant. The curves were then summed and are compared with the normalized photon curve in Fig. 4(a). The agreement between 
the normalized x-ray curve and the photon fraction arising from DR, (estimated from the TOF results) is remarkably good up to the KLM resonances. This is a bit surprising as polarization effects (as the $\mathrm{x}$ rays are measured at $90^{\circ}$ ) and some possible higher-order decays, such as $2 \mathrm{E} 1$, were ignored. An explanation could be that $\mathrm{Si}$ has a rather low nuclear charge number [33], and we sum in the x-ray spectrum over several transitions where polarization effects average out. The excellent fit of the summed DR allows us to extract a EIE rate coefficient spectrum, normalized to H-like $\mathrm{Si}$ and containing the He-like and other EIE curves weighted by the abundance ratios of various charge states with respect to $\mathrm{H}$-like Si. The obtained excitation curve shows strong peaks associated with resonant EIE [see Fig. 4(b)].

\section{SUMMARY AND CONCLUSION}

To summarize, we studied electron-ion collisions in an EBIT by accumulating a charge-changing process, detecting them by TOF, and simultaneously measuring the photons from the trapped Si ions. Monitoring the evolution of all individual charge states and emitted photons from Si provides selective access to electron-impact recombination and excitation processes. The data were analyzed to get absolute charge-state separated DR rate coefficients for H-like, He-like, and Li-like Si. A comparison of some resonance groups of the DR of XIV and XIII Si with state-of-the-art RMBPT calculations shows excellent agreement in energy and resonance strength. Although the TOF measurement gives information about the charge-state-separated DR, the x-ray spectra contain photons emitted both during recombination and as a result of EIE followed by photon emission. Therefore, through a subtraction of the TOF from the X-ray spectra, it is possible to identify and separate the EIE process, which produces photons without changing the charge state of the ions. This allows us to give the electron-impact excitation rates of highly charged $\mathrm{Si}$ ions besides the recombination rates that are needed for plasma modeling.

\section{ACKNOWLEDGMENTS}

We thank S. Böhm and T. Fritioff for experimental assistance, F. Ferro for assistance with the theoretical data, and D. Savin for fruitful discussions. Financial support from the Wallenberg Foundation and the Swedish Research Council (VR) is gratefully acknowledged.
[1] C. W. Danforth and J. M. Shull, Astrophys. J. 679, 194 (2008).

[2] C. Day, Phys. Today 61(1), 19 (2008).

[3] A. Müller, Adv. At., Mol., Opt. Phys. 55, 293 (2008).

[4] K. M. Aggarwal, K. Hamada, A. Igarashi, V. Jonauskas, F. P. Keenan, and S. Nakazaki, Astron. Astrophys. 484, 879 (2008).

[5] S. Chantrenne, P. Beiersdorfer, R. Cauble, and M. B. Schneider, Phys. Rev. Lett. 69, 265 (1992).

[6] C. Shah, J. R. Crespo López-Urrutia, M. Feng Gu, T. Pfeifer, J. Marques, F. Grilo, J. P. Santos, and P. Amaro, Astrophys. J. 881, 100 (2019).

[7] M. Piwiński et al., J. Phys. B: At., Mol. Opt. Phys. 51, 085002 (2018).

[8] R. Schuch and S. Böhm, J. Phys.: Conf. Ser. 88, 012002 (2007).

[9] R. E. Marrs, M. A. Levine, D. A. Knapp, and J. R. Henderson, Phys. Rev. Lett. 60, 1715 (1988).

[10] I. Orban, P. Glans, Z. Altun, E. Lindroth, A. Källberg, and R. Schuch, Astron. Astrophys. 459, 291 (2006).

[11] I. Orban, E. Lindroth, P. Glans, and R. Schuch, J. Phys. B: At., Mol. Opt. Phys. 40, 1063 (2007).

[12] I. Orban, S. D. Loch, S. Böhm, and R. Schuch, Astrophys. J. 721, 1603 (2010).

[13] T. M. Baumann, Z. Harman, J. Stark, C. Beilmann, G. Liang, P. H. Mokler, J. Ullrich, and J. R. Crespo López-Urrutia, Phys. Rev. A 90, 052704 (2014).

[14] R. Ali, C. P. Bhalla, C. L. Cocke, and M. Stockli, Phys. Rev. Lett. 64, 633 (1990).

[15] D. R. DeWitt, D. Schneider, M. W. Clark, M. H. Chen, and D. Church, Phys. Rev. A 44, 7185 (1991).

[16] N. Nakamura, H. Tobiyama, H. Nohara, D. Kato, H. Watanabe, F. J. Currell, and S. Ohtani, Phys. Rev. A 73, 020705(R) (2006).

[17] S. Böhm et al., J. Phys.: Conf. Ser. 58, 303 (2007).
[18] S. Mahmood, S. Ali, I. Orban, S. Tashenov, E. Lindroth, and R, Schuch, Astrophys. J. 754, 86 (2012).

[19] D. H. Zhang, Z. W. Wu, C. Ren, J. Jiang, L. Y. Xie, R. Schuch, J. M. Zhang, and C. Z. Dong, Astrophys. J., Suppl. Ser. 247, 22 (2020).

[20] D. R. DeWitt, E. Lindroth, R. Schuch, H. Gao, T. Quinteros, and W. Zong, J. Phys. B: At., Mol. Opt. Phys. 28, L147 (1995).

[21] M. Tokman et al., Phys. Rev. A 66, 012703 (2002).

[22] M. Hobein., I. Orban, S. Böhm, A. Solders, M. Suhonen, T. Fritioff, S. Tashenov, and R. Schuch, J. Instrum. 5, C11003 (2010).

[23] H. Watanabe, H. Tobiyama, A. P. Kavanagh, Y. M. Li, N. Nakamura, H. A. Sakaue, F. J. Currell, and S. Ohtani, Phys. Rev. A 75, 012702 (2007).

[24] B. M. Penetrante, J. N. Bardsley, D. DeWitt, M. Clark, and D. Schneider, Phys. Rev. A 43, 4861 (1991).

[25] X. Lu and F. J. Currell, Phys. Rev. Spec. Top.-Accel. Beams 12, 014401 (2009).

[26] M. Stobbe, Ann. Phys. (NY) 7, 661 (1930).

[27] D. J. McLaughlin and Y. Hahn, Phys. Rev. A 43, 1313 (1991).

[28] P. Bryans, N. R. Badnell, T. W. Gorczyca, J. M. Laming, W. Mitthumsiri, and D. W. Savin, Astrophys. J., Suppl. Ser. 167, 343 (2006).

[29] K. P. Dere, Astron. Astrophys. 466, 771 (2007).

[30] M. A. Lennon, K. L. Bell, H. B. Gilbody, J. G. Hughes, A. E. Kingston, M. J. Murray, and F. J. Smith, J. Phys. Chem. Ref. Data 17, 1285 (1988).

[31] R. Clark and J. Abdallah, Jr., IAEA-APID 11, 1 (2003).

[32] J. V. Porto, I. Kink, and J. D. Gillaspy, Rev. Sci. Instrum. 71, 3050 (2000).

[33] Z.-W. Wu, J. Jiang, and C.-Z. Dong, Phys. Rev. A 84, 032713 (2011). 